\title{
Robot-assisted minimally invasive esophagectomy versus video-assisted minimally invasive esophagectomy: a systematic review and meta-analysis
}

\author{
Hao Chen ${ }^{1 \#} \wedge$, Yiyang Liu ${ }^{1 \#}$, Hao Peng ${ }^{2}$, Rongchun Wang ${ }^{2}$, Kang Wang ${ }^{1}$, Demin Li ${ }^{1}$ \\ ${ }^{1}$ Department of Cardiothoracic Surgery, Jinling Hospital, Jinling School of Clinical Medicine, Nanjing Medical University, Nanjing, China; \\ ${ }^{2}$ Department of Cardiothoracic Surgery, Affiliated Jinling Hospital, Medical School of Nanjing University, Nanjing, China \\ Contributions: (I) Conception and design: H Chen, Y Liu, D Li; (II) Administrative support: D Li, K Wang; (III) Provision of study materials or \\ patients: H Chen, R Wang; (IV) Collection and assembly of data: H Chen, Y Liu; (V) Data analysis and interpretation: H Chen, H Peng; (VI) \\ Manuscript writing: All authors; (VII) Final approval of manuscript: All authors. \\ \#These authors contributed equally to this work. \\ Correspondence to: Demin Li, MD, PhD; Kang Wang, MD. Department of Cardiothoracic Surgery, Jinling Hospital, Jinling School of Clinical Medicine, \\ Nanjing Medical University, 305 East Zhongshan Road, Xuanwu District, Nanjing 210002, China. Email: dr.demin@126.com; lw37413@sina.com.
}

\begin{abstract}
Background: Robot-assisted minimally invasive esophagectomy (RAMIE) has been demonstrated to offer realistic three-dimensional visual clarity, flexible movement and so on. The high cost is the main reason hampering universal application. The aim of this study was to compare the short-term outcomes of RAMIE versus video-assisted minimally invasive esophagectomy (VAMIE).

Methods: The PubMed, EMBASE and Web of Science databases were systematically searched up to June 1, 2021, for studies comparing RAMIE and VAMIE.

Results: Nineteen studies were enrolled, which consisted of a total of 4,714 patients, including 2,306 patients in the RAMIE group and 2,408 patients in the VAMIE group. In RAMIE patients, higher numbers of total lymph nodes ( $\mathrm{MD}=0.171,95 \% \mathrm{CI}: 0.086-0.255, \mathrm{P}<0.001)$ and lymph nodes along the left recurrent laryngeal nerve $(\mathrm{RLN})(\mathrm{MD}=0.219,95 \% \mathrm{CI}: 0.097-0.340, \mathrm{P}<0.001)$ were removed. In RAMIE patients in the McKown group, higher numbers of total lymph nodes (MD =0.173, 95\% CI: $0.080-0.265, \mathrm{P}<0.001)$ and lymph nodes along the left RLN (MD =0.220, 95\% CI: 0.090-0.350, P=0.001) were removed, while in those in the ESCC group, higher numbers of total lymph nodes (MD =0.249, 95\% CI: 0.091-0.407, P=0.002) and lymph nodes along the left RLN (MD =0.239, 95\% CI: $0.102-0.377, \mathrm{P}=0.001)$ were removed.

Discussion: This study indicated that the main advantage of RAMIE was a greater number of harvested lymph nodes, which may be beneficial to diagnosis and local control. RCTs with larger sample sizes and studies reporting long-term outcomes are needed to evaluate the advantages and disadvantages of RAMIE and VAMIE.
\end{abstract}

Keywords: Robot-assisted minimally invasive esophagectomy (RAMIE); video-assisted minimally invasive esophagectomy (VAMIE); meta-analysis; short-term outcomes

Submitted Aug 02, 2021. Accepted for publication Oct 14, 2021.

doi: $10.21037 /$ tcr-21-1482

View this article at: https://dx.doi.org/10.21037/tcr-21-1482

\footnotetext{
^ ORCID: 0000-0001-9683-8278.
} 


\section{Introduction}

Esophageal cancer is a gastrointestinal tumor that ranks seventh in terms of incidence and sixth in mortality (1). Although chemotherapy, radiotherapy and immunotherapy have been widely used in clinical practice, esophagectomy is still the primary treatment for patients with esophageal cancer. Video-assisted minimally invasive esophagectomy (VAMIE) has become increasingly prevalent due to the lower incidence of postoperative complications and shorter hospital stay compared with conventional open esophagectomy (2). However, VAMIE still has some limitations. Recently, the Da Vinci surgical system has been introduced, with advantages including realistic threedimensional visual clarity, flexible movement and so on. This system can filter out tremors and synchronize with surgeons' movements to a certain extent. Nevertheless, the high costs and the lack of haptic feedback are the main disadvantages impeding universal application. The safety and feasibility of robot-assisted minimally invasive esophagectomy (RAMIE) have been confirmed (3). Although RAMIE has been demonstrated to offer better visualization and enable meticulous dissection of the mediastinum structure and lymph nodes, the actual superiorities of RAMIE over VAMIE have not been verified. To date, two meta-analyses $(4,5)$ are available for reporting the comparison between RAMIE and VAMIE. They reached an agreement with a lower incidence of vocal cord palsy in RAMIE and were inconsistent in other aspects. More high-quality studies have been published, and we investigated the actual advantages of RAMIE over VAMIE according to short-term outcomes. We present the following article in accordance with the PRISMA reporting checklist (available at https://dx.doi. org/10.21037/tcr-21-1482).

\section{Methods}

\section{Literature search}

The PubMed, EMBASE and Web of Science databases were searched from 1980 to June 1, 2021. The following search terms were used: ((((Esophageal Neoplasms) OR (Esophageal Neoplasm)) OR (Neoplasm, Esophageal)) OR (Esophagus Neoplasm)) OR ((Esophagectomy) OR (Esophagectomies))) AND ((((Robotic Surgical Procedures) OR (Procedure, Robotic Surgical)) OR (Procedures, Robotic Surgical)) OR (Robotic Surgical Procedure))) AND ((()(Thoracoscopy) OR (Thoracoscopies Pleural
Endoscopy)) OR (Pleuroscopy)) OR (Pleuroscopies)) OR (((((Thoracic Surgery, Video-Assisted) OR (Surgeries, Video-Assisted Thoracic)) OR (Surgery, Video-Assisted Thoracic)) OR (Thoracic Surgeries, Video-Assisted))).

\section{Study selection}

This meta-analysis enrolled randomized controlled trials (RCTs) and retrospective cohort studies (RCSs) comparing RAMIE and VAMIE in terms of short-term clinical outcomes. The following studies were excluded: case reports or reviews, studies on other topics such as the feasibility of RAMIE and articles with overlapping patients.

\section{Outcomes of interest}

The outcomes of interest consisted of the numbers of total lymph nodes, thoracic lymph nodes, and lymph nodes along the left recurrent laryngeal nerve (RLN) and right RLN, RLN paresis, anastomotic leakage, chylothorax, pneumonia, operative time (min), blood loss (mL), length of stay (LOS), 30-day mortality and 90-day mortality.

\section{Quality assessment}

The selected studies included 18 RCSs and 1 RCT, and the NOS and Cochrane Library were used for grading according to the types of studies. The risk of bias assessment was carried out by two reviewers (H Chen and Y Liu) independently. A third reviewer (H Peng) arbitrated disagreements.

\section{Statistical analysis}

The meta-analysis was performed using Stata 12.0. Continuous variables are presented as the standard mean difference (SMD) with a 95\% confidence interval (CI). Dichotomous variables were pooled using risk ratios (RRs) with $95 \%$ CIs. Heterogeneity was evaluated by the $\mathrm{c}^{2}-$ based test and $\mathrm{P}$ values. If high heterogeneity was observed $\left(\mathrm{I}^{2}>50 \%\right)$, the random-effect model was used; otherwise, the fixed-effect model was used. Forest plots were created to show the pooled estimates for the studies. Subgroup analysis and sensitivity analysis were used to investigate the source of heterogeneity. Publication bias was assessed using Begg's test. P values $<0.05$ were considered statistically significant. 


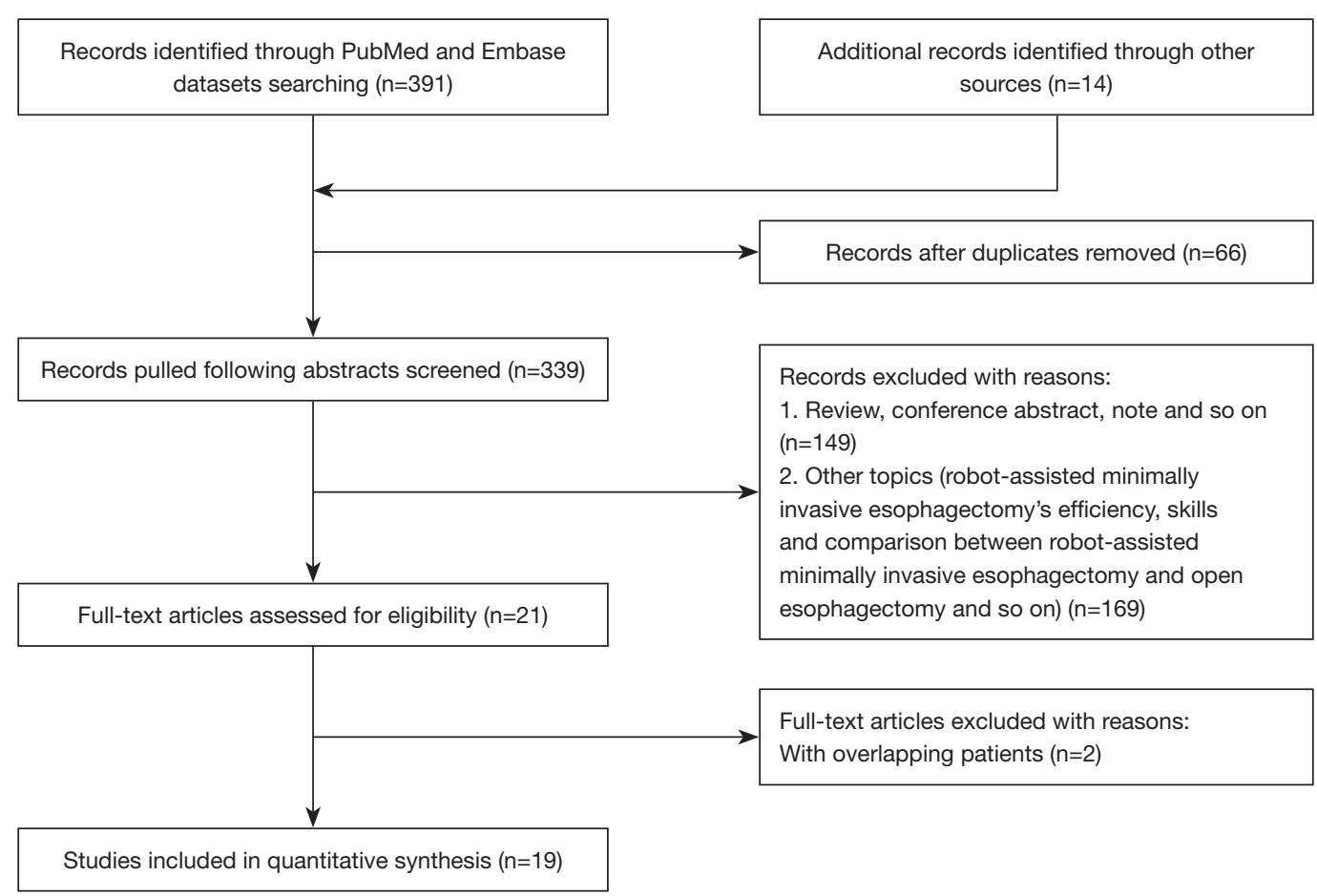

Figure 1 Flow chart of study selection.

\section{Results}

\section{Basic characteristics}

A total of 391 articles were found in PubMed and EMBASE (Figure 1). Another 14 articles were added after identification through other sources. After removing 66 duplicates, 339 articles remained. After reviewing the titles and abstracts, 318 articles were excluded because they did not fulfill the inclusion criteria. The remaining 21 articles were further assessed for eligibility by examining the full text. Finally, 19 relevant studies (6-24) were included in this meta-analysis. Two studies $(25,26)$ were excluded due to data duplication. The treatment center published more than one article with overlapping patients. The final sample consisted of 4,714 patients, 2,306 of whom were subjected to RAMIE, while 2,408 were subjected to VAMIE. The basic characteristics of the included studies are presented in Table 1. All the incidence rates and mean values in the included studies are shown in Table 2. The RCSs were of high quality based on the NOS. The methodological quality assessment scores are summarized in Figure 2. One study was an RCT, and the quality assessment was high based on Cochrane analysis.

\section{Number of harvested lymph nodes}

The number of total lymph nodes excised was described in 12 studies, the number of thoracic lymph nodes was described in 4 studies, and the number of lymph nodes along the left and right RLNs was described in 4 studies. The meta-analysis indicated that in RAMIE patients, higher numbers of total lymph nodes ( $M D=0.171,95 \% \mathrm{CI}$ : 0.086-0.255, $\mathrm{P}<0.001)$ and lymph nodes along the left RLN ( $\mathrm{MD}=0.219,95 \% \mathrm{CI}: 0.097-0.340, \mathrm{P}<0.001)$ were removed (Figure 3). Begg's test was conducted to assess publication bias, and no publication bias was found for the number of total lymph nodes $(\mathrm{P}=0.732)$ or the number of lymph nodes along the left RLN ( $\mathrm{P}=0.308)$. The differences between RAMIE and VAMIE were not statistically significant for other aspects.

\section{Postoperative complications}

RLN paresis was reported in 14 studies, anastomotic leakage was reported in 15 studies, and chylothorax was reported in 13 studies. Pooled data analysis showed that the differences in RLN paresis, anastomotic leakage and 
Table 1 Characteristics of the selected studies included in the meta-analysis

\begin{tabular}{|c|c|c|c|c|c|c|c|c|c|c|c|c|c|}
\hline Study & Year & Country & Group & Number & Gender (M/F) & Age, years & BMI, kg/m² & Site (upper/mid/lower) & Pathology (ESCC/EAC) & Neoadjuvant therapy & Surgical procedures & Type of study & NOS \\
\hline \multirow[t]{2}{*}{ Suda } & 2012 & Japan & RAMIE & 16 & $15 / 1$ & 65 (53 to 86 ) & $21.3(17.5$ to 26.3$)$ & $2 / 7 / 7$ & $16 / 0$ & 6 & NA & RCS & 7 \\
\hline & & & VAMIE & 20 & $15 / 5$ & 64.5 (50 to 79 ) & 20.4 (14.9 to 24.8$)$ & 2/12/6 & $20 / 0$ & 17 & NA & & \\
\hline \multirow[t]{2}{*}{ Weksler } & 2012 & America & RAMIE & 11 & $8 / 3$ & $58.7 \pm 8.5$ & 27.1 & NA & 0/10 & 4 & Ivor-Lewis & RCS & 7 \\
\hline & & & VAMIE & 26 & $20 / 6$ & $64.3 \pm 11.3$ & 27.9 & NA & $3 / 23$ & 10 & Ivor-Lewis & & \\
\hline \multirow[t]{2}{*}{ Park } & 2016 & South Korea & RAMIE & 62 & $57 / 5$ & $64.3 \pm 8.0$ & $23.5 \pm 2.8$ & $8 / 15 / 39$ & $62 / 0$ & 8 & Both & RCS & 8 \\
\hline & & & VAMIE & 43 & $40 / 3$ & $66.2 \pm 7.4$ & $23.3 \pm 3.1$ & $7 / 9 / 27$ & $43 / 0$ & 4 & Both & & \\
\hline \multirow[t]{2}{*}{ Yerokun } & 2016 & America & RAMIE & 170 & $142 / 28$ & $56 / 64 / 70$ & NA & $156 / 14 / 0$ & NA & 120 & NA & RCS & 7 \\
\hline & & & VAMIE & 170 & $143 / 27$ & $56 / 63 / 69$ & NA & $160 / 10 / 0$ & NA & 120 & NA & & \\
\hline \multirow[t]{2}{*}{ Weksler } & 2017 & America & RAMIE & 569 & $471 / 98$ & $62.9 \pm 9.6$ & NA & NA & $\mathrm{NA} / 447$ & 405 & NA & RCS & 8 \\
\hline & & & VAMIE & 569 & $489 / 80$ & $62.8 \pm 9.3$ & NA & NA & $\mathrm{NA} / 468$ & 401 & NA & & \\
\hline \multirow[t]{2}{*}{$\mathrm{He}$} & 2018 & China & RAMIE & 27 & $20 / 7$ & $61.0 \pm 8.0$ & $21.5 \pm 2.7$ & $1 / 18 / 8$ & $23 / \mathrm{NA}$ & 0 & McKown & RCS & 8 \\
\hline & & & VAMIE & 27 & $20 / 7$ & $61.6 \pm 9.8$ & $21.9 \pm 2.8$ & $3 / 15 / 9$ & $25 / \mathrm{NA}$ & 0 & Mckown & & \\
\hline \multirow[t]{2}{*}{ Deng } & 2018 & China & RAMIE & 52 & $40 / 12$ & $61.0 \pm 7.2$ & NA & $10 / 33 / 9$ & $52 / 0$ & NA & McKown & RCS & 8 \\
\hline & & & VAMIE & 52 & $39 / 13$ & $60.9 \pm 9.2$ & NA & $7 / 30 / 14$ & $52 / 0$ & NA & Mckown & & \\
\hline \multirow[t]{2}{*}{ Grimminger } & 2018 & Germany & RAMIE & 25 & $22 / 3$ & $61.1 \pm 11.1$ & $25.6 \pm 4.3$ & $0 / 2 / 23$ & $7 / 18$ & 9 & Ivor-Lewis & RCS & 7 \\
\hline & & & VAMIE & 25 & $19 / 6$ & $63 \pm 8.7$ & $25.5 \pm 4.5$ & $0 / 5 / 20$ & $9 / 16$ & 7 & Ivor-Lewis & & \\
\hline \multirow[t]{2}{*}{ Chen } & 2019 & China & RAMIE & 54 & $41 / 13$ & $61.8 \pm 9.4$ & $22.7 \pm 2.9$ & NA & $54 / 0$ & 14 & McKown & RCS & 8 \\
\hline & & & VAMIE & 54 & $43 / 11$ & $61.8 \pm 8.3$ & $23.0 \pm 2.7$ & NA & $54 / 0$ & 17 & McKown & & \\
\hline \multirow[t]{2}{*}{ Espinoza-Mercado } & 2019 & America & RAMIE & 406 & NA & NA & NA & NA & NA & NA & NA & RCS & 8 \\
\hline & & & VAMIE & 406 & NA & NA & NA & NA & NA & NA & NA & & \\
\hline \multirow[t]{2}{*}{ Motoyama } & 2019 & Japan & RAMIE & 21 & $19 / 2$ & $63(44-76)$ & NA & $6 / 7 / 8$ & $21 / 0$ & 0 & NA & RCS & 7 \\
\hline & & & VAMIE & 38 & $32 / 6$ & $66(49-75)$ & NA & $9 / 16 / 13$ & $38 / 0$ & 1 & NA & & \\
\hline \multirow[t]{2}{*}{ Washington } & 2019 & America & RAMIE & 18 & $17 / 1$ & $61.9(42-76)$ & $27.6(20.7-38.2)$ & NA & $\mathrm{NA} / 14$ & 18 & McKown & RCS & 8 \\
\hline & & & VAMIE & 18 & $16 / 2$ & $58.9(40-70)$ & $27.5(19.2-39.4)$ & NA & $\mathrm{NA} / 15$ & 15 & Mckown & & \\
\hline \multirow[t]{2}{*}{ Zhang } & 2019 & China & RAMIE & 66 & $50 / 16$ & $62.3 \pm 7.8$ & $22.9 \pm 3.1$ & 0/29/37 & $64 / 0$ & NA & Ivor-Lewis & RCS & 8 \\
\hline & & & VAMIE & 66 & $50 / 16$ & $62.0 \pm 7.8$ & $23.1 \pm 4.5$ & $0 / 26 / 40$ & $65 / 0$ & NA & Ivor-Lewis & & \\
\hline \multirow[t]{2}{*}{ Chao } & 2020 & America & RAMIE & 39 & $35 / 4$ & $57.41 \pm 8.59$ & $22.35 \pm 2.76$ & $12 / 16 / 11$ & $38 / 1$ & 39 & Mckown & RCS & 8 \\
\hline & & & VAMIE & 67 & $65 / 2$ & $54.55 \pm 7.93$ & $22.34 \pm 3.25$ & $12 / 35 / 20$ & $65 / 2$ & 67 & McKown & & \\
\hline \multirow[t]{2}{*}{ Gong } & 2020 & China & RAMIE & 91 & $78 / 13$ & 60.04 & NA & $7 / 31 / 53$ & $86 / \mathrm{NA}$ & 20 & McKown & RCS & 8 \\
\hline & & & VAMIE & 144 & $130 / 14$ & 60.22 & NA & $4 / 72 / 68$ & $134 / \mathrm{NA}$ & 28 & McKown & & \\
\hline \multirow[t]{2}{*}{ Xu } & 2020 & China & RAMIE & 292 & $220 / 72$ & $64.34 \pm 8.25$ & $22.96 \pm 2.95$ & $20 / 214 / 58$ & $292 / 0$ & NA & McKown & RCS & 8 \\
\hline & & & VAMIE & 292 & $216 / 76$ & $64.91 \pm 7.99$ & $23.21 \pm 3.19$ & $24 / 214 / 54$ & $292 / 0$ & NA & McKown & & \\
\hline \multirow[t]{2}{*}{ Yang } & 2020 & China & RAMIE & 271 & $222 / 49$ & $63.4 \pm 7.1$ & $23.2 \pm 3.0$ & $38 / 169 / 64$ & $271 / 0$ & 29 & McKown & RCS & 8 \\
\hline & & & VAMIE & 271 & $221 / 50$ & $63.5 \pm 7.4$ & $23.2 \pm 2.9$ & $31 / 171 / 69$ & $270 / 0$ & 28 & McKown & & \\
\hline \multirow[t]{2}{*}{$\mathrm{He}$} & 2020 & China & RAMIE & 94 & $72 / 22$ & $61.3 \pm 8.2$ & $22.7 \pm 2.8$ & $9 / 64 / 21$ & $94 / 0$ & 0 & Mckown & $\mathrm{RCT}$ & \\
\hline & & & VAMIE & 98 & $72 / 76$ & $62.4 \pm 9.1$ & $22.8 \pm 3.0$ & $7 / 68 / 23$ & $98 / 0$ & 0 & McKown & & \\
\hline \multirow[t]{2}{*}{ Balasubramanian } & 2021 & England & RAMIE & 22 & $14 / 8$ & $60.91 \pm 9.31$ & $19.07 \pm 2.08$ & $0 / 6 / 6$ & $17 / 5$ & 17 & Both & RCS & 8 \\
\hline & & & VAMIE & 22 & $12 / 10$ & $59.27 \pm 11.60$ & $18.91 \pm 2.07$ & $1 / 5 / 4$ & $17 / 5$ & 19 & Both & & \\
\hline
\end{tabular}

NA, not available, the enrolled studies did not show the result; RAMIE, robot-assisted 


\begin{tabular}{|c|c|c|c|c|c|c|c|c|c|c|c|c|c|c|c|}
\hline Study & Group & $\mathrm{N}$ & $\begin{array}{c}\text { Total lymph } \\
\text { nodes dissected }\end{array}$ & $\begin{array}{l}\text { Thoracic lymph } \\
\text { nodes dissected }\end{array}$ & $\begin{array}{l}\text { Lymph nodes dissected } \\
\text { along left RLN }\end{array}$ & $\begin{array}{l}\text { Lymph nodes dissected } \\
\text { along right RLN }\end{array}$ & RLN paresis & $\begin{array}{l}\text { Anastomotic } \\
\text { leak }\end{array}$ & Chylothorax & Pneumonia & Operative time & Blood loss & LOS & $\begin{array}{l}\text { 30-day } \\
\text { mortality }\end{array}$ & $\begin{array}{l}\text { 90-day } \\
\text { mortality }\end{array}$ \\
\hline \multirow[t]{2}{*}{ Suda } & RAMIE & 16 & 37.5 (23 to 63 ) & 18.5 (11 to 39$)$ & $5.5(0$ to 13$)$ & NA & 9 & 6 & 0 & 1 & NA & NA & 22 (7 to 67$)$ & 0 & 0 \\
\hline & VAMIE & 20 & 39 (24 to 63$)$ & 22.5 (13 to 41$)$ & 6.5 (0 to 14$)$ & NA & 17 & 2 & 2 & 4 & NA & NA & 35.5 (20 to 135$)$ & 0 & 0 \\
\hline \multirow[t]{2}{*}{ Weksler } & RAMIE & 11 & $23 \pm 10$ & NA & NA & NA & 1 & 1 & NA & 1 & NA & NA & $8.7 \pm 3.4$ & NA & NA \\
\hline & VAMIE & 26 & $23 \pm 10$ & NA & NA & NA & 1 & 4 & NA & 4 & NA & NA & $10.0 \pm 7.7$ & NA & NA \\
\hline \multirow[t]{2}{*}{ Park } & RAMIE & 62 & $37.3 \pm 17.1$ & NA & NA & NA & 8 & 5 & NA & NA & $490.3 \pm 84.0$ & $462.9 \pm 493.9$ & NA & 1 & NA \\
\hline & VAMIE & 43 & $28.7 \pm 11.8$ & NA & NA & NA & 10 & 1 & NA & NA & $458.4 \pm 111.9$ & $466.8 \pm 333.0$ & NA & 0 & NA \\
\hline \multirow[t]{2}{*}{ Yerokun } & RAMIE & 170 & $11 / 16 / 21$ & NA & NA & NA & NA & NA & NA & NA & NA & NA & $8 / 10 / 14$ & 10 & NA \\
\hline & VAMIE & 170 & $11 / 16 / 22$ & NA & NA & NA & NA & NA & NA & NA & NA & NA & $8 / 10 / 13$ & 15 & NA \\
\hline \multirow[t]{2}{*}{ Weksler } & RAMIE & 569 & 16.0 (10.0 to 23.0$)$ & NA & NA & NA & NA & NA & NA & NA & $349 \pm 45$ & $119 \pm 72$ & NA & 32 & 46 \\
\hline & VAMIE & 569 & 16.0 (10.0 to 23.0$)$ & NA & NA & NA & NA & NA & NA & NA & $285 \pm 66$ & $158 \pm 82$ & NA & 56 & 39 \\
\hline \multirow[t]{2}{*}{$\mathrm{He}$} & RAMIE & 27 & $20 \pm 7$ & NA & NA & NA & 4 & 3 & 0 & 5 & NA & NA & $13.8 \pm 2.0$ & NA & 0 \\
\hline & VAMIE & 27 & $19 \pm 5$ & NA & NA & NA & 3 & 1 & 1 & 2 & NA & NA & $12.8 \pm 2.7$ & NA & 1 \\
\hline \multirow[t]{2}{*}{ Deng } & RAMIE & 52 & $21.5 \pm 8.4$ & $11.8 \pm 5.1$ & $1.0 \pm 1.8$ & $2.4 \pm 1.9$ & 7 & 3 & 0 & 5 & $353.0 \pm 71.8$ & NA & $14.3 \pm 6.9$ & NA & 2 \\
\hline & VAMIE & 52 & $17.3 \pm 6.5$ & $10.1 \pm 4.3$ & $0.4 \pm 0.8$ & $1.9 \pm 2.2$ & 4 & 2 & 1 & 4 & $274.2 \pm 51.7$ & NA & $12.7 \pm 7.7$ & NA & 2 \\
\hline \multirow[t]{2}{*}{ Grimminger } & RAMIE & 25 & $24.5 \pm 11.4$ & NA & NA & NA & NA & 3 & 1 & 2 & $410.2 \pm 75.1$ & NA & $21.8 \pm 18.1$ & 0 & 1 \\
\hline & VAMIE & 25 & $25.0 \pm 9.4$ & NA & NA & NA & NA & 4 & 0 & 3 & $338.8 \pm 52.1$ & NA & $17.2 \pm 11.9$ & 0 & 0 \\
\hline \multirow[t]{2}{*}{ Chen } & RAMIE & 54 & $25.4 \pm 7.5$ & NA & NA & NA & 7 & 5 & 1 & 8 & $187.2 \pm 34.0$ & NA & $17.1 \pm 10.1$ & 0 & NA \\
\hline & VAMIE & 54 & $24.7 \pm 11.2$ & NA & NA & NA & 17 & 2 & 2 & 13 & $193.4 \pm 27.1$ & NA & $15.2 \pm 9.8$ & 0 & NA \\
\hline \multirow[t]{2}{*}{ Espinoza-Mercado } & RAMIE & 406 & 17 (11 to 24$)$ & NA & NA & NA & NA & NA & NA & NA & NA & NA & NA & 16 & 31 \\
\hline & VAMIE & 406 & 16 (10 to 22$)$ & NA & NA & NA & NA & NA & NA & NA & NA & NA & NA & 13 & 25 \\
\hline \multirow[t]{2}{*}{ Motoyama } & RAMIE & 21 & 52 (36 to 104$)$ & 23 (11 to 41$)$ & $6(0$ to 15$)$ & NA & 7 & 1 & 1 & 0 & 634 (529 to 699) & NA & NA & NA & NA \\
\hline & VAMIE & 38 & 59 (35 to 97$)$ & 20 (7 to 68$)$ & $4(0$ to 12$)$ & NA & 30 & 3 & 1 & 0 & 598.5 (475 to 761$)$ & NA & NA & NA & NA \\
\hline \multirow[t]{2}{*}{ Washington } & RAMIE & 18 & 14.28 (4 to 30$)$ & NA & NA & NA & NA & 1 & NA & NA & 168 (127 to 212) & NA & 9.9 (7 to 20$)$ & NA & NA \\
\hline & VAMIE & 18 & 13.9 (2 to 28$)$ & NA & NA & NA & NA & 1 & NA & NA & 164 (135 to 25 to 228$)$ & NA & 9.8 (7 to 27$)$ & NA & NA \\
\hline \multirow[t]{2}{*}{ Zhang } & RAMIE & 66 & $19.2 \pm 9.2$ & $10.3 \pm 5.8$ & $1.3 \pm 1.9$ & $1.4 \pm 1.6$ & 4 & 5 & 0 & 4 & NA & 200.0 (100.0 to 262.5 ) & $9.0(8.0$ to 12.3$)$ & 0 & 1 \\
\hline & VAMIE & 66 & $19.3 \pm 9.5$ & $11.9 \pm 8.3$ & $0.9 \pm 1.9$ & $1.6 \pm 2.8$ & 3 & 3 & 1 & 5 & NA & 200.0 (150.0 to 245.0 ) & $9.0(8.0$ to 11.3$)$ & 0 & 1 \\
\hline \multirow[t]{2}{*}{ Chao } & RAMIE & 39 & 29 (26 to 33) & 14 (10 to 17$)$ & $3(1$ to 5$)$ & 1 (1 to 2$)$ & 4 & NA & 0 & 1 & NA & NA & NA & 0 & 0 \\
\hline & VAMIE & 67 & 28 (21 to 35$)$ & 14 (10 to 17$)$ & $1(0$ to 4$)$ & $2(1$ to 3$)$ & 19 & NA & 2 & 11 & NA & NA & NA & 0 & 3 \\
\hline \multirow[t]{2}{*}{ Gong } & RAMIE & 91 & $22.84 \pm 8.37$ & NA & $2.35 \pm 3.00$ & $2.74 \pm 2.03$ & 20 & 4 & 1 & 9 & $318.02 \pm 53.90$ & $215.49 \pm 125.40$ & $16.57 \pm 8.00$ & NA & 0 \\
\hline & VAMIE & 144 & $23.07 \pm 10.18$ & NA & $1.95 \pm 2.67$ & $2.57 \pm 2.08$ & 34 & 10 & 1 & 15 & $321.13 \pm 57.21$ & $200.49 \pm 59.54$ & $18.73 \pm 13.29$ & NA & 0 \\
\hline \multirow[t]{2}{*}{$\mathrm{xu}$} & RAMIE & 292 & $21.83 \pm 7.73$ & $12.60 \pm 4.22$ & $2.27 \pm 0.90$ & $3.06 \pm 1.05$ & 24 & 21 & 3 & 25 & NA & NA & NA & NA & 4 \\
\hline & VAMIE & 292 & $20.85 \pm 4.73$ & $11.83 \pm 3.12$ & $2.09 \pm 0.79$ & $2.97 \pm 1.08$ & 27 & 24 & 4 & 29 & NA & NA & NA & NA & 4 \\
\hline \multirow[t]{2}{*}{ Yang } & RAMIE & 271 & $20.3 \pm 9.9$ & $12.4 \pm 7.0$ & NA & NA & 79 & 32 & 4 & 24 & $244.5 \pm 60.4$ & $210.7 \pm 86.8$ & 11 (6 to 54$)$ & NA & 0 \\
\hline & VAMIE & 271 & $19.2 \pm 9.6$ & $12.4 \pm 6.5$ & NA & NA & 41 & 39 & 2 & 34 & $276.0 \pm 59.4$ & $209.6 \pm 107.4$ & 11 (4 to 94$)$ & NA & 2 \\
\hline \multirow[t]{2}{*}{$\mathrm{He}$} & RAMIE & 94 & $29.2 \pm 12.5$ & NA & NA & NA & 6 & 7 & 2 & 6 & $304.2 \pm 82.5$ & $202.5 \pm 73.4$ & $12(5$ to 78$)$ & 2 & NA \\
\hline & VAMIE & 98 & $22.8 \pm 13.3$ & NA & NA & NA & 9 & 9 & 2 & 9 & $315.5 \pm 35.7$ & $216.8 \pm 44.6$ & 13 (8 to 125$)$ & 1 & NA \\
\hline \multirow[t]{2}{*}{ Balasubramanian } & RAMIE & 22 & $23.95 \pm 8.23$ & NA & NA & NA & 3 & 0 & 1 & 3 & $513.18 \pm 91.23$ & $138.86 \pm 31.2$ & $12.18 \pm 6.35$ & NA & NA \\
\hline & VAMIE & 22 & $22.73 \pm 11.63$ & NA & NA & NA & 1 & 1 & 1 & 4 & $444.77 \pm 64.91$ & $133.18 \pm 34.8$ & $12.73 \pm 7.83$ & NA & NA \\
\hline
\end{tabular}




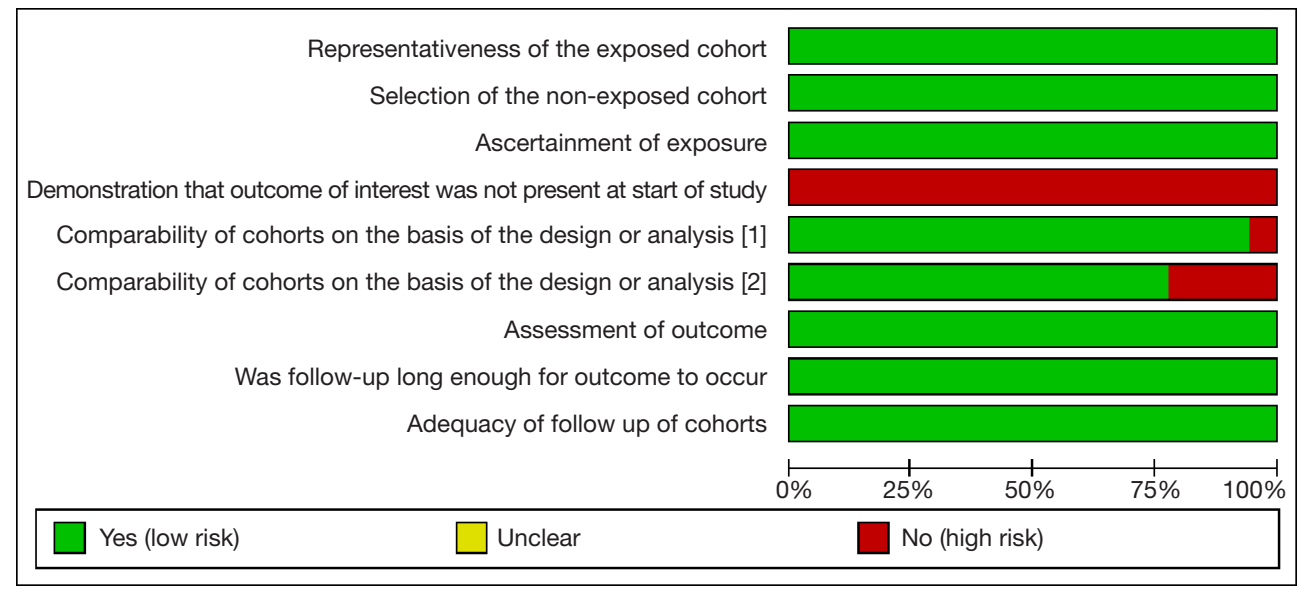

Figure 2 The Newcastle-Ottawa scale.

chylothorax were not statistically significant (Figure 4). Pneumonia was reported in 14 studies. One study did not divide patients into RAMIE and VAMIE groups and was therefore excluded. In 2 studies, RAMIE patients developed pneumonia more frequently than VAMIE patients. Moreover, VAMIE patients had a higher pneumonia incidence in 11 studies. Pooled data analysis showed that RAMIE was associated with a lower incidence of pneumonia $(\mathrm{RR}=0.842$, 95\% CI: 0.716-0.989, $\mathrm{P}=0.036$ ).

\section{Operative time, blood loss and LOS}

A total of 9 studies reported the operative time. In 5 studies, RAMIE patients had longer operative times than VAMIE patients, while VAMIE patients had longer operative times in 4 studies. The meta-analysis showed no significant difference between RAMIE and VAMIE $(\mathrm{P}=0.161)$. Blood loss was reported in 6 studies, and LOS was reported in 7 studies. No significant differences in blood loss and LOS were found between these two techniques (Figure 5).

\section{Mortality after the operation}

The 30-day mortality rate was reported in 5 studies, and the 90-day mortality rate was reported in 9 studies. As shown in Figure 6, pooled data analysis showed that differences were not statistically significant for 30-day mortality $(\mathrm{RR}=0.826,95 \% \mathrm{CI}: 0.680-1.003, \mathrm{P}=0.053$ ) or 90-day mortality (RR $=1.059,95 \% \mathrm{CI}: 0.911-1.231$,
$\mathrm{P}=0.457)$.

\section{Subgroup analysis}

Summary of the meta-analysis results are shown in Table 3. According to surgical methods, patients were assigned to the McKown group in 9 studies and to the Ivor-Lewis group in 3 studies. In the McKown group, higher numbers of total lymph nodes (MD $=0.173,95 \%$ CI: $0.080-0.265$, $\mathrm{P}<0.001)$ and lymph nodes along the left RLN $(\mathrm{MD}=0.220$, 95\% CI: 0.090-0.350, $\mathrm{P}=0.001)$ were removed in RAMIE patients than in VAMIE patients. No significant difference was found between RAMIE and VAMIE in the Ivor-Lewis group. Based on pathology, patients were diagnosed with esophageal squamous cell carcinoma (ESCC) in 9 studies, while esophageal adenocarcinoma (EAC) was not diagnosed in any studies. In the ESCC group, higher numbers of total lymph nodes ( $\mathrm{MD}=0.249,95 \% \mathrm{CI}$ : $0.091-0.407, \mathrm{P}=0.002$ ) and lymph nodes along the left RLN (MD $=0.239,95 \%$ CI: $0.102-0.377, \mathrm{P}=0.001)$ were removed in the RAMIE patients (Figure 7).

\section{Discussion}

Esophageal cancer causes a serious global health burden, and esophagectomy is considered the primary treatment. Traditional open esophagectomy has been gradually replaced by VAMIE. Some RCTs (27-29) have shown that VAMIE is associated with a lower incidence of postoperative complications and a better prognosis than open esophagectomy. Over the past decade, the Da Vinci 
A Study$$
\text { ID }
$$

Weksler, B. (2012)

Park, S. (2016)

He, H. (2018)

Deng, H. Y. (2018)

Grimminger, P. P. (2018)

Chen, J (2019)

Zhang, Y. (2019)

Gong, L. (2020)

$\mathrm{Xu}, \mathrm{Y},(2020)$

Yang, Y. (2020)

He, Z. F. (2020)

Balasubramanian (2021)

Overall (l-squared $=36.5 \%, p=0.098$ )

$-964$

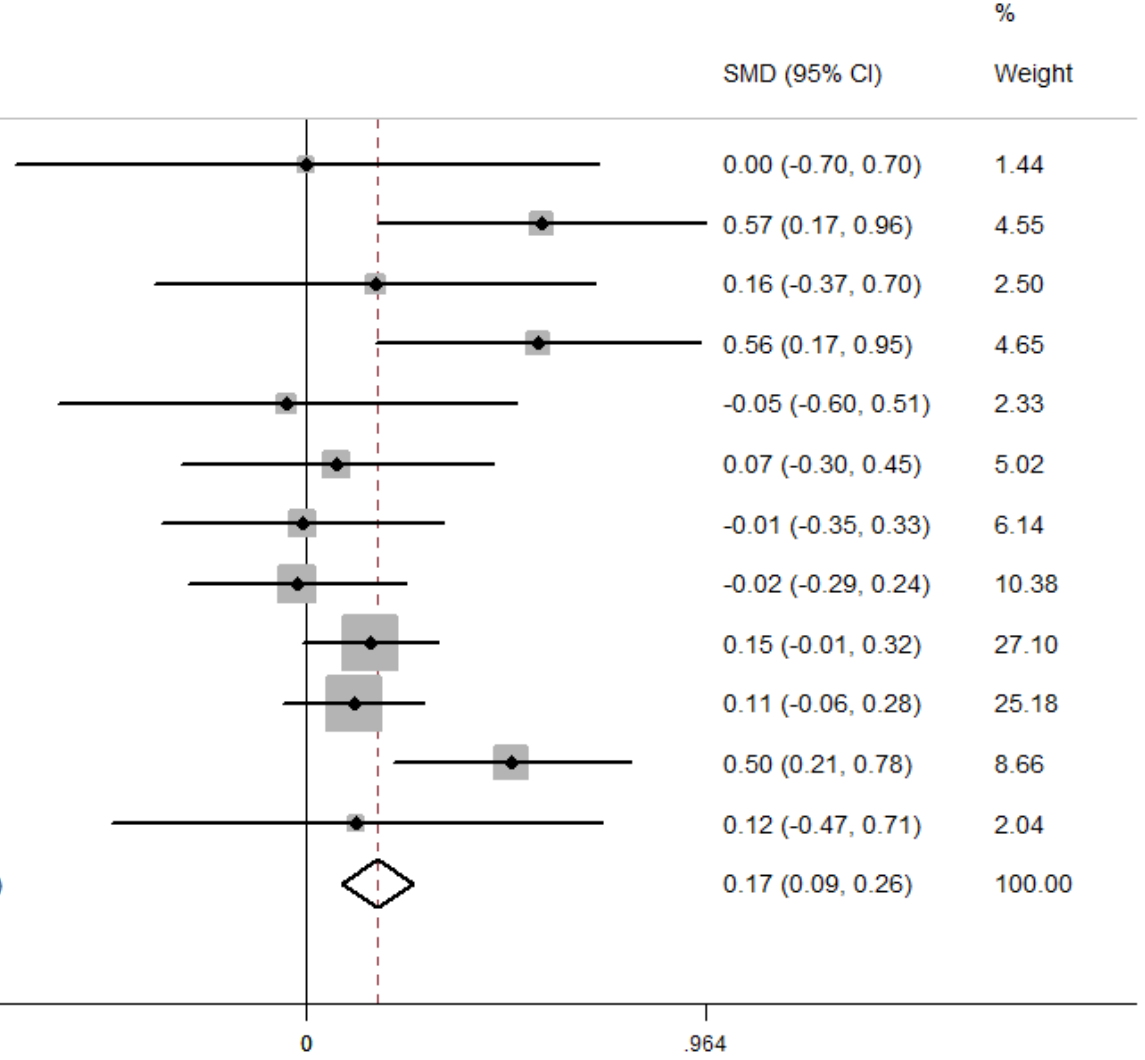

C study

ID

$\operatorname{SMD}(95 \%$ Cl)

\section{$\%$}

Deng, H. Y. (2018)

Zhang, Y. (2019)

Gong, L. (2020)

$\mathrm{Xu,} \mathrm{Y.} \mathrm{(2020)}$

Overall (1-squared $=0.0 \%, p=0.689$ )
$0.21(-0.13,0.55) \quad 12.67$

$0.14(-0.12,0.41) \quad 21.48$

$0.21(0.05,0.38) \quad 56.05$

$0.22(0.10,0.34) \quad 100.00$

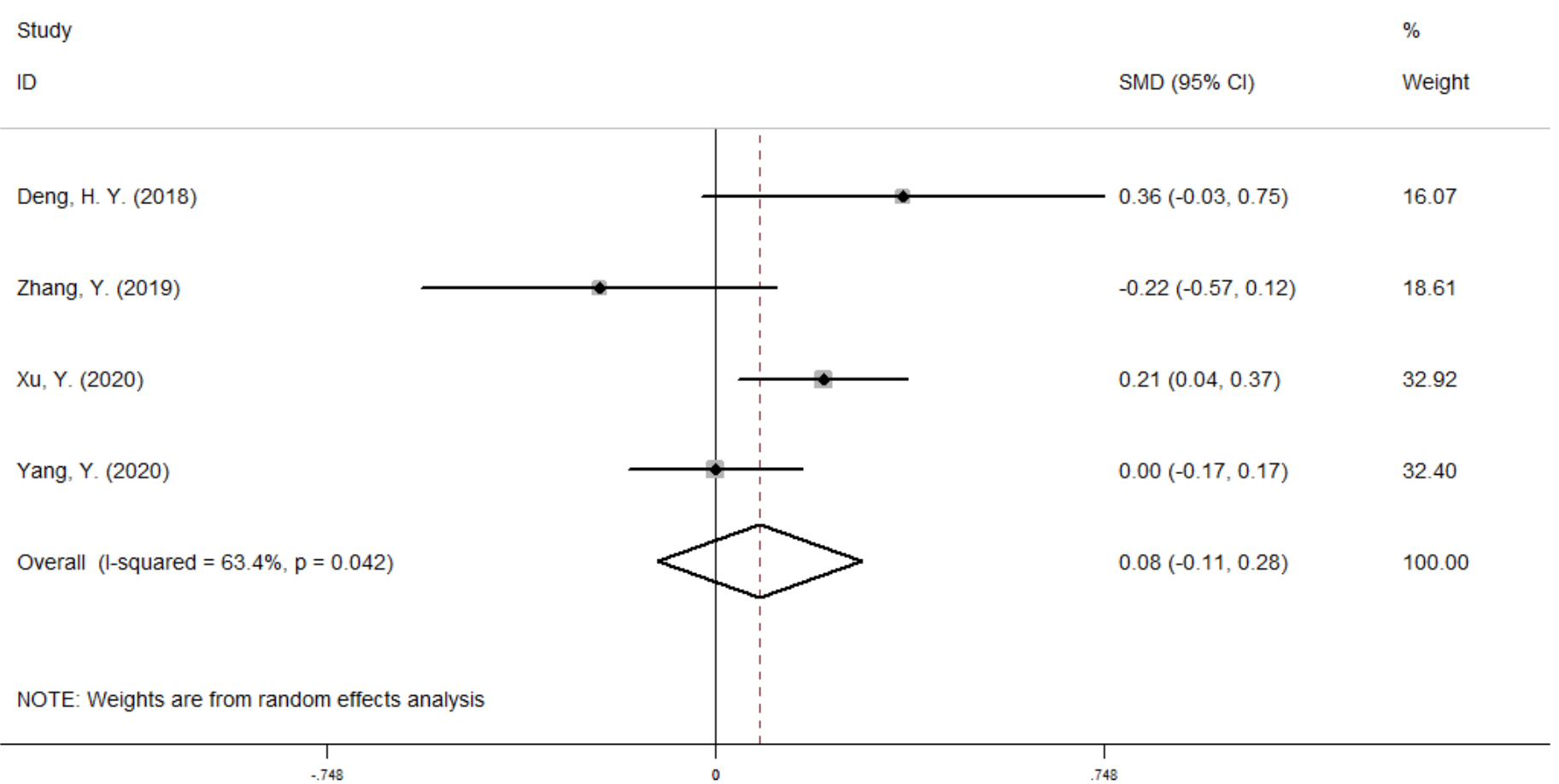

D study

\begin{tabular}{|c|c|c|}
\hline ID & SMD $(95 \% \mathrm{Cl})$ & Weight \\
\hline Deng, H. Y. (2018) & $0.24(-0.14,0.63)$ & 9.91 \\
\hline Zhang, Y. (2019) & $-0.09(-0.43,0.25)$ & 12.66 \\
\hline Gong, L. (2020) & $0.08(-0.18,0.35)$ & 21.40 \\
\hline $\mathrm{Xu}, \mathrm{Y} .(2020)$ & $0.08(-0.08,0.25)$ & 56.03 \\
\hline Overall (I-squared $=0.0 \%, p=0.655$ ) & $0.08(-0.04,0.20)$ & 100.00 \\
\hline
\end{tabular}

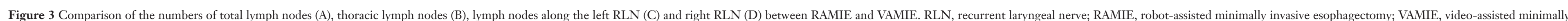
invasive esophagectomy. 
A study

Suda, K. (2012) Weksler, B. (2012) Park, S. (2016)

He, H. (2018) Deng, H. Y. (2018) Chen, J (2019) Motoyama, S. (2019) Zhang, Y. (2019) Chao, Y. K. (2019)

Gong, L. (2020)

$\mathrm{Xu,}, \mathrm{Y} .(2020)$

Yang, Y. (2020)

He, Z. F. (2020)

Balasubramanian (2021)

Overall (I-squared $=72.9 \%, \mathrm{p}=0.000$

NOTE: Weights are from random effects analys

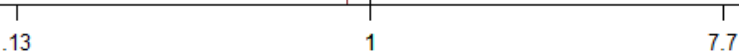

C stud

ID

Suda, K. (2012)

He, H. (2018)

Deng, H. Y. (2018)

Grimminger, P. P. (2018)

Chen, J (2019)

Motoyama, S. (2019)

Zhang, Y. (2019)

Chao, Y. K. (2019)

Gong, L. (2020)

$\mathrm{Xu}, \mathrm{Y} .(2020)$

Yang. Y. (2020)

He, Z. F. (2020)

Balasubramanian (2021)

Overall (l-squared $=0.0 \%, p=0.925$ )

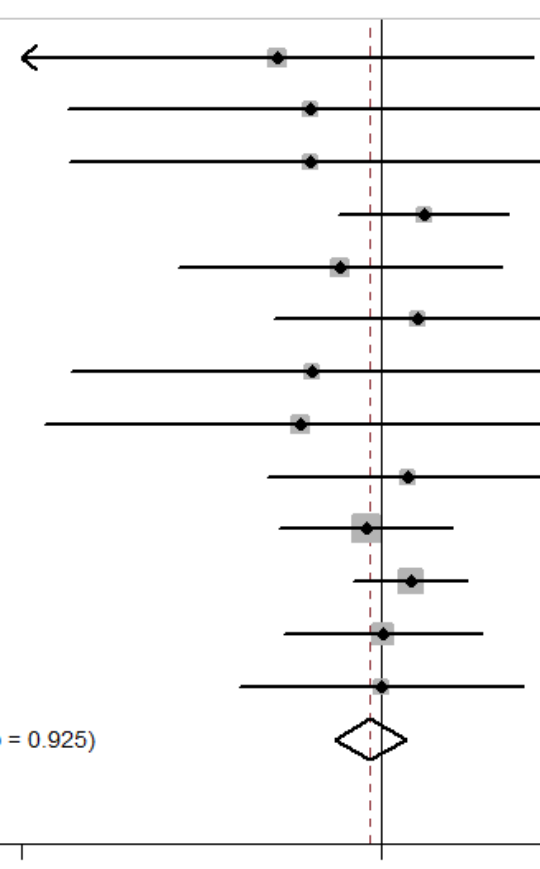

$\int_{0.1}^{275}$

$\operatorname{RR~}(95 \%$ Cl) Weigh

$0.49(0.25,0.96) \quad 6.67$

$\begin{array}{ll}1.75(0.40,7.70) & 2.48 \\ 0.72(0.42,123) & 7.79\end{array}$

$1.17(0.58,2.36) \quad 6.34$

$132(0.80 .215) \quad 826$

$\begin{array}{ll}1.32(0.80,2.15) & 8.26 \\ 0.52(0.27,1.00) & 6.79\end{array}$

$0.30(0.14,0.62) \quad 6.0$

$1.15(0.59,2.24) \quad 6.67$

$0.41(0.16,1.04) \quad 4.77$

$0.94(0.64,1.40) \quad 9.25$

$0.94(0.69,1.27) \quad 10.08$

$145(1.23,1.71) \quad 11.12$

$0.80(0.43,1.52) \quad 6.92$

$1.58(0.82,3.03) \quad 6.78$

$0.88(0.67,1.14) \quad 100.00$

17

$\%$

$0.35(0.03,4.55) \quad 7.04$

$0.49(0.04,5.49) \quad 5.30$

$0.50(0.04,5.50) \quad 5.35$

$1.53(0.66,3.58) \quad 5.09$

$0.66(0.13,3.31) \quad 7.95$

$1.43(0.34,5.96) \quad 3.66$

$0.50(0.04,5.51) \quad 5.36$

$0.44(0.03,5.63) \quad 5.93$

$1.29(0.32,5.22) \quad 4.1$

$0.86(0.36,2.02) \quad 18.71$

$1.34(0.76,2.37) \quad 15.96$

$1.02(0.38,2.75) \quad 10.35$

$1.00(0.24,4.13) \quad 5.16$

$0.90(0.63,1.29) \quad 100.00$
B study

ID

Suda, K. (2012)

Weksler, B. (2012)

Park, S. (2016)

He, H. (2018)

Deng, H. Y. (2018)

Grimminger, P. P. (2018)

Chen, J (2019)

Motoyama, S. (2019)

Washington, K. (2019)

Zhang, Y. (2019)

Gong, L. (2020)

$\mathrm{Xu}, \mathrm{Y} .(2020)$

Yang, Y. (2020)

He, Z. F. (2020)

Balasubramanian (2021)

Overall (1-squared $=15.9 \%, p=0.27$

-

.0436

RR $(95 \%$ Cl) Weigh

$2.10(1.11,3.97) \quad 2.50$

$0.64(0.10,3.98) \quad 1.52$

$1.45(0.97,2.15) \quad 3.67$

$1.56(0.83,2.95) \quad 2.00$

$1.21(0.58,2.55) \quad 2.65$

$0.84(0.34,2.07) \quad 3.47$

$1.47(0.88,2.45) \quad 3.58$

$0.69(0.12,3.89) \quad 1.53$

$100(024,416) \quad 106$

$1.27(0.72,2.24) \quad 4.16$

$0.73(0.31,1.69) \quad 5.84$

$0.93(0.67,1.28) \quad 23.51$

$0.89(0.68,1.17) \quad 35.25$

$0.89(0.50,1.57) \quad 8.16$

$0.49(0.04,5.49) \quad 1.10$

$0.99(0.85,1.15) \quad 100.00$

D Study

ID

Suda, K. (2012)

Weksler, B. (2012)

He, H. (2018)

Deng, H. Y. (2018)

Grimminger, P. P. (2018)

Chen, J (2019)

Zhang, Y. (2019)

Chao, Y. K. (2019)

Gong, L. (2020)

$\mathrm{Xu,} \mathrm{Y.} \mathrm{(2020)}$

Yang, Y. (2020)

He, Z. F. (2020)

Balasubramanian (2021)

Motoyama, S. (2019)

Overall (--squared $=0.0 \%, p=0.707$ )

,

0311

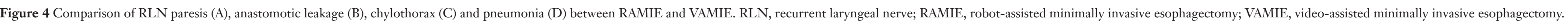




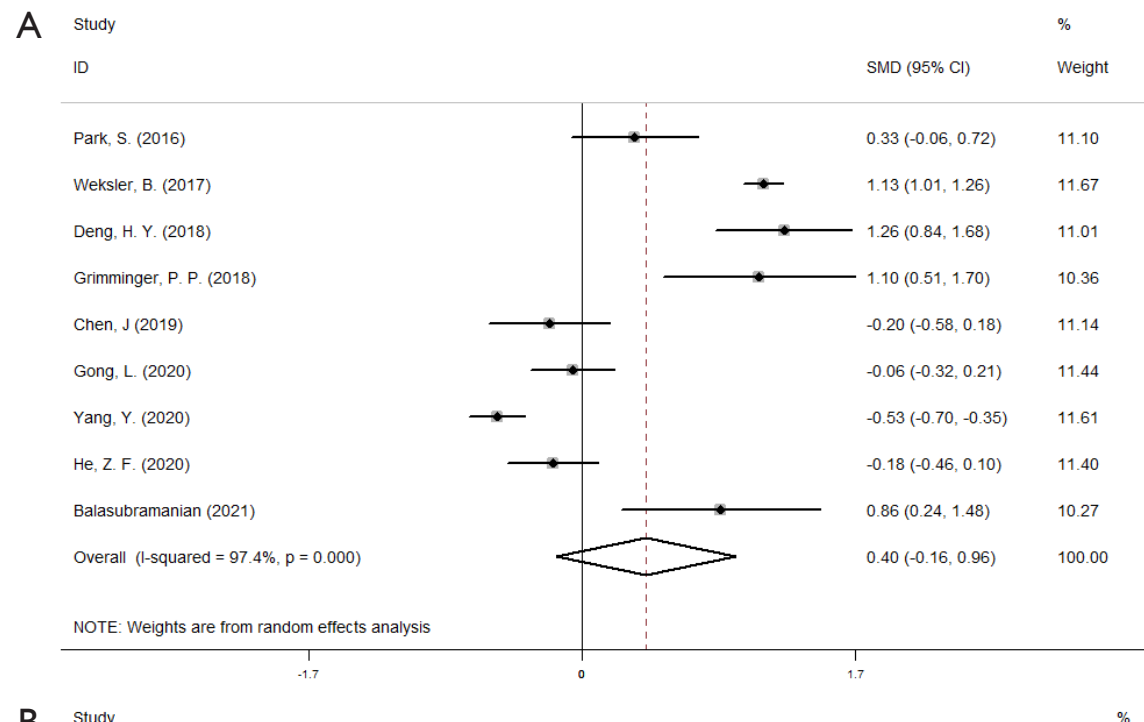

B

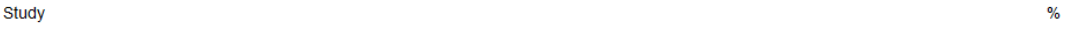

D.

Park, S. (2016)

Weksler, B. (2017)

Gong, L. (2020)

Yang, Y. (2020)

He, Z. F. (2020)

Balasubramanian (2021)

Overall (1-squared $=87.6 \%, p=0.000)$

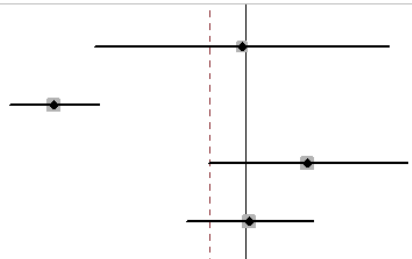

$-0.01(-0.40,0.38) \quad 14.84$

$-0.51(-0.62,-0.39) \quad 20.18$

$0.17(-0.10,0.43) \quad 17.63$

$0.01(-0.16,0.18) \quad 19.45$

$-0.24(-0.52,0.05) \quad 17.18$

$0.17(-0.42,0.76)-10.72$

$-0.09(-0.37,0.18) \quad 100.00$

NOTE: Weights are from random effects analysis

$\underset{-.764}{1}$

C study

$\operatorname{SMD}(95 \% \mathrm{Cl})$

Weight

Weksler, B. (2012)

He, H. (2018)

Deng, H. Y. (2018)

Grimminger, P. P. (2018)

Chen, J (2019)

Gong, L. (2020)

Balasubramanian (2021)

Overall (l-squared $=21.2 \%, p=0.268$ )

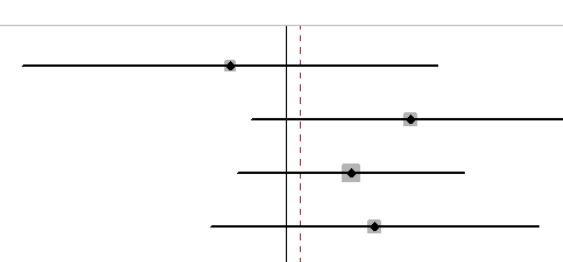

$-0.19(-0.90,0.51) \quad 5.05$

$0.42(-0.12,0.96) \quad 8.65$

$0.22(-0.17,0.60) \quad 16.95$

$0.30(-0.26,0.86)$

$0.19(-0.19,0.57) \quad 17.62$

$-0.19(-0.45,0.08) \quad 36.42$

$-0.08(-0.67,0.51) \quad 7.21$

$0.05(-0.11,0.21) \quad 100.00$

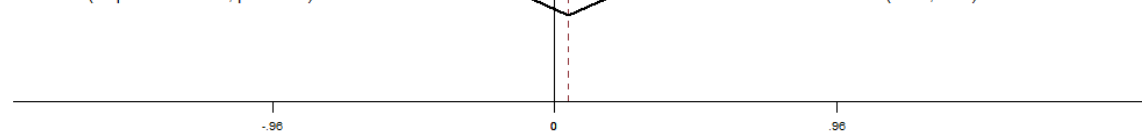

Figure 5 Comparison of operative time (A), blood loss (B) and LOS (C) between RAMIE and VAMIE. LOS, length of stay; RAMIE, robotassisted minimally invasive esophagectomy; VAMIE, video-assisted minimally invasive esophagectomy. 
A Study

ID

$\operatorname{RR}(95 \% \mathrm{Cl}) \quad$ Weight

Park, S. (2016)

Yerokun, B. A. (2016)

Weksler, B. (2017)

Espinoza-Mercado, F. (2019)

He, Z. F. (2020)

Suda, K. (2012)

Grimminger, P. P. (2018)

Chen, J (2019)

Zhang, Y. (2019)

Chao, Y. K. (2019)

Overall (l-squared $=39.8 \%, p=0.156)$

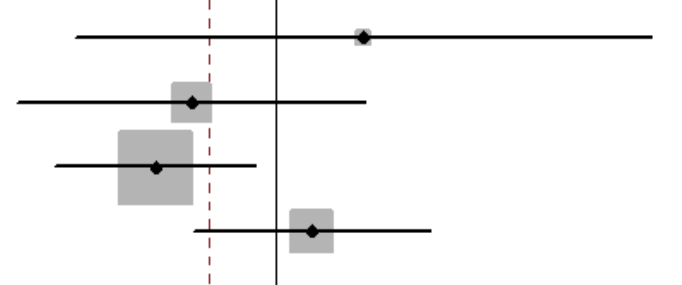

$1.28(0.57,2.90) \quad 1.65$

$0.79(0.48,1.29) \quad 16.85$

$0.71(0.54,0.94) \quad 59.49$

$1.11(0.79,1.55) \quad 19.95$

$1.37(0.61,3.09) \quad 2.06$

(Excluded) $\quad 0.00$

(Excluded) $\quad 0.00$

(Excluded) $\quad 0.00$

(Excluded) $\quad 0.00$

(Excluded) $\quad 0.00$

$0.83(0.68,1.00) \quad 100.00$

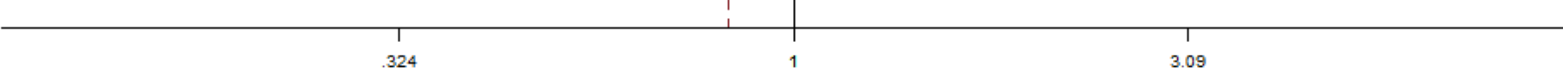

B Study

ID

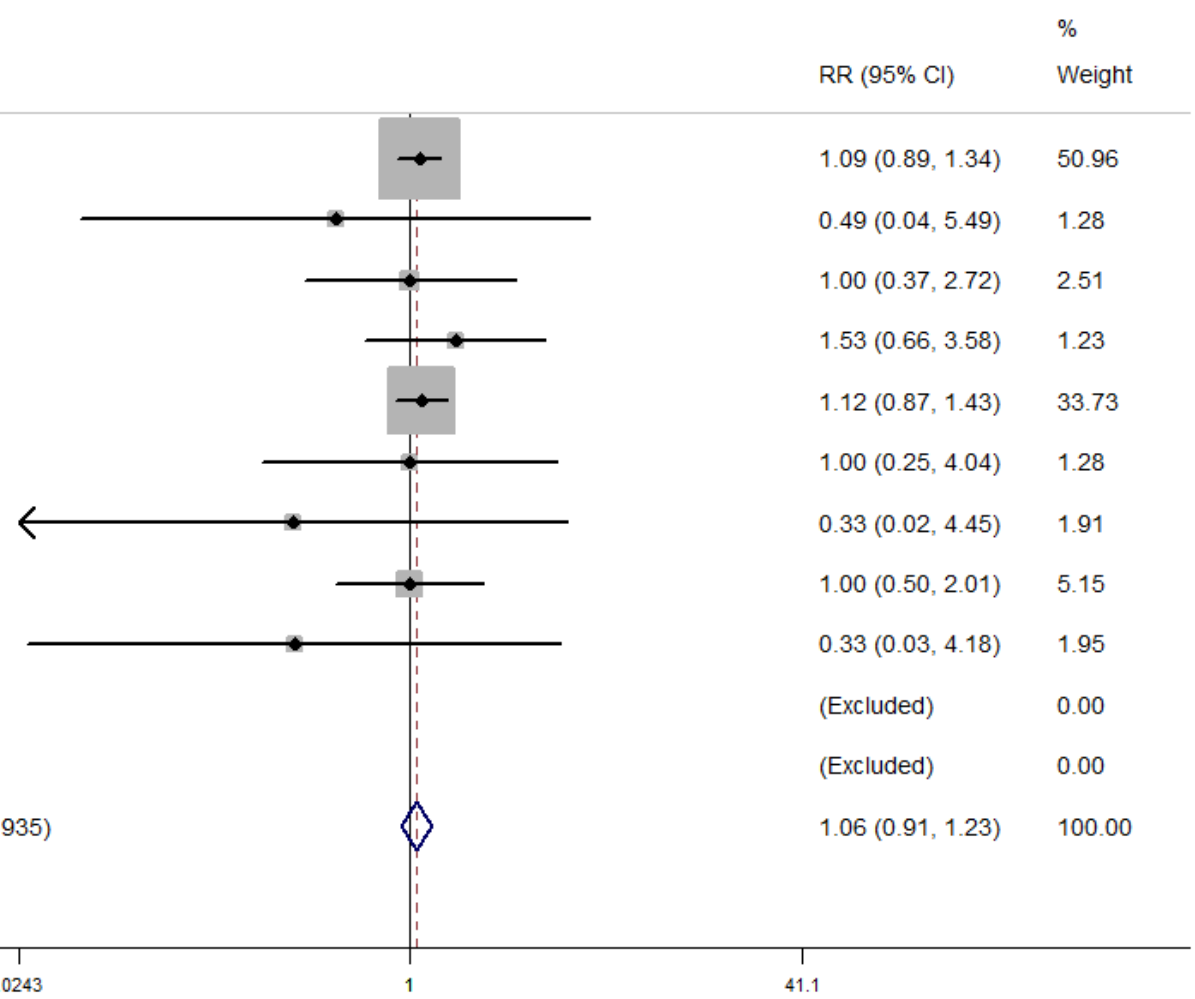

Figure 6 Comparison of 30-day mortality (A) and 90-day mortality (B) between RAMIE and VAMIE. RAMIE, robot-assisted minimally invasive esophagectomy; VAMIE, video-assisted minimally invasive esophagectomy. 
Table 3 Summary of the meta-analysis results

\begin{tabular}{|c|c|c|c|c|c|c|c|c|}
\hline \multirow{2}{*}{ Outcomes of interest } & \multirow{2}{*}{ Number } & \multirow{2}{*}{ Inference } & \multicolumn{2}{|c|}{ Fixed-effects model } & \multicolumn{2}{|c|}{ Random-effects model } & \multicolumn{2}{|c|}{ Heterogeneity } \\
\hline & & & SMD/RR (95\% Cl) & $\mathrm{P}$ & RR $(95 \% \mathrm{Cl})$ & $\mathrm{P}$ & $1^{2}$ & $\mathrm{P}$ \\
\hline \multicolumn{9}{|l|}{ Total } \\
\hline Total lymph nodes & 12 & $7,8,11,12,13,14,17,20,21,22,23,24$ & $0.171(0.086,0.255)$ & 0.000 & $0.185(0.066,0.304)$ & 0.002 & $36.50 \%$ & 0.098 \\
\hline Thoracic lymph nodes & 4 & $12,18,21,22$ & $0.094(-0.012,0.201)$ & 0.082 & $0.085(-0.112,0.282)$ & 0.400 & $63.40 \%$ & 0.042 \\
\hline Lymph nodes along the left RLN & 4 & $12,18,20,21$ & $0.219(0.097,0.340)$ & 0.000 & $0.219(0.097,0.340)$ & 0.000 & $0.00 \%$ & 0.689 \\
\hline Lymph nodes along the right RLN & 4 & $12,18,20,21$ & $0.078(-0.043,0.199)$ & 0.208 & $0.078(-0.043,0.199)$ & 0.208 & $0.00 \%$ & 0.655 \\
\hline RLN paresis & 14 & $6,7,8,11,12,14,16,18,19,20,21,22,23,24$ & $0.988(0.880,1.110)$ & 0.844 & $0.876(0.673,1.140)$ & 0.324 & $72.90 \%$ & 0.000 \\
\hline Anastomotic leak & 15 & $6,7,8,11,12,13,14,16,17,18,20,21,22,23,24$ & $0.986(0.848,1.146)$ & 0.852 & $1.110(0.940,1.312)$ & 0.219 & $15.90 \%$ & 0.275 \\
\hline Chylothorax & 13 & $6,11,12,13,14,16,18,19,20,21,22,23,24$ & $0.897(0.625,1.288)$ & 0.558 & $1.086(0.786,1.502)$ & 0.617 & $0.00 \%$ & 0.925 \\
\hline Pneumonia & 13 & $6,7,11,12,13,14,18,19,20,21,22,23,24$ & $0.842(0.716,0.989)$ & 0.036 & $0.895(0.765,1.048)$ & 0.167 & $0.00 \%$ & 0.707 \\
\hline Blood loss & 6 & $8,10,20,22,23,24$ & $-0.251(-0.335,-0.168)$ & 0.000 & $-0.094(-0.372,0.183)$ & 0.506 & $87.60 \%$ & 0.000 \\
\hline LOS & 7 & $7,11,12,13,14,20,24$ & $0.048(-0.111,0.207)$ & 0.553 & $0.072(-0.116,0.260)$ & 0.453 & $21.20 \%$ & 0.268 \\
\hline 30-day mortality & 5 & $8,9,10,15,23$ & $0.826(0.680,1.003)$ & 0.053 & $0.923(0.706,1.209)$ & 0.562 & $39.80 \%$ & 0.156 \\
\hline 90-day mortality & 9 & $10,11,12,13,15,18,19,21,22$ & $1.059(0.911,1.231)$ & 0.457 & $1.091(0.941,1.265)$ & 0.248 & $0.00 \%$ & 0.935 \\
\hline \multicolumn{9}{|l|}{ McKown } \\
\hline Total lymph nodes & 7 & $11,12,14,20,21,22,23$ & $0.173(0.080,0.265)$ & 0.000 & $0.195(0.053,0.338)$ & 0.007 & $48.10 \%$ & 0.073 \\
\hline Thoracic lymph nodes & 3 & $12,21,22$ & $0.128(0.016,0.240)$ & 0.025 & $0.148(-0.038,0.333)$ & 0.118 & $55.70 \%$ & 0.104 \\
\hline Lymph nodes along the left RLN & 3 & $12,20,21$ & $0.220(0.090,0.350)$ & 0.001 & $0.220(0.090,0.350)$ & 0.001 & $0.00 \%$ & 0.001 \\
\hline Lymph nodes along the right RLN & 3 & $12,20,21$ & $0.102(-0.028,0.232)$ & 0.124 & $0.102(-0.028,0.232)$ & 0.124 & $0.00 \%$ & 0.748 \\
\hline RLN paresis & 8 & $11,12,14,19,20,21,22,23$ & $1.068(0.940,1.213)$ & 0.315 & $0.956(0.717,1.275)$ & 0.760 & $71.10 \%$ & 0.001 \\
\hline Anastomotic leak & 8 & $11,12,14,19,20,21,22,23$ & $0.942(0.793,1.118)$ & 0.492 & $0.995(0.842,1.175)$ & 0.950 & $0.00 \%$ & 0.489 \\
\hline Pneumonia & 8 & $11,12,14,19,20,21,22,23$ & $0.855(0.721,1.014)$ & 0.072 & $0.912(0.758,1.098)$ & 0.332 & $11.80 \%$ & 0.339 \\
\hline Operative time & 5 & $12,14,20,22,23$ & $-0.207(-0.324,-0.091)$ & 0.000 & $0.038(-0.452,0.528)$ & 0.879 & $93.50 \%$ & 0.000 \\
\hline Blood loss & 3 & $20,22,23$ & $-0.002(-0.129,0.124)$ & 0.971 & $-0.011(-0.207,0.185)$ & 0.916 & $52.40 \%$ & 0.123 \\
\hline LOS & 4 & $11,12,19,20$ & $0.049(-0.129,0.227)$ & 0.590 & $0.109(-0.161,0.379)$ & 0.430 & $51.70 \%$ & 0.102 \\
\hline 30-day mortality & 1 & 23 & $1.370(0.607,3.089)$ & 0.449 & - & - & - & - \\
\hline 90-day mortality & 5 & $11,12,19,21,22$ & $0.747(0.421,1.325)$ & 0.318 & $0.878(0.516,1.496)$ & 0.633 & $0.00 \%$ & 0.756 \\
\hline \multicolumn{9}{|l|}{ ESCC } \\
\hline Total lymph nodes & 7 & $8,12,14,18,21,22,23$ & $0.206(0.112,0.300)$ & 0.000 & $0.249(0.091,0.407)$ & 0.002 & $56.60 \%$ & 0.032 \\
\hline Thoracic lymph nodes & 4 & $12,18,21,22$ & $0.094(-0.012,0.201)$ & 0.082 & $0.085(-0.112,0.282)$ & 0.400 & $63.40 \%$ & 0.042 \\
\hline Lymph nodes along the left RLN & 3 & $12,18,21$ & $0.239(0.102,0.377)$ & 0.001 & $0.239(0.102,0.377)$ & 0.001 & $0.00 \%$ & 0.588 \\
\hline Lymph nodes along the right RLN & 3 & $12,18,21$ & $0.077(-0.060,0.214)$ & 0.272 & $0.077(-0.060,0.214)$ & 0.272 & $0.00 \%$ & 0.446 \\
\hline RLN paresis & 9 & $6,8,12,14,16,18,21,22,23$ & $1.016(0.896,1.151)$ & 0.806 & $0.812(0.578,1.140)$ & 0.229 & $80.10 \%$ & 0.000 \\
\hline Anastomotic leak & 9 & $6,8,12,14,16,18,21,22,23$ & $1.009(0.860,1.182)$ & 0.917 & $1.148(0.932,1.413)$ & 0.195 & $37.20 \%$ & 0.121 \\
\hline Chylothorax & 8 & $6,12,14,16,18,21,22,23$ & $0.890(0.585,1.354)$ & 0.587 & $1.047(0.714,1.536)$ & 0.814 & $0.00 \%$ & 0.831 \\
\hline Pneumonia & 7 & $6,12,14,16,18,21,22$ & $0.844(0.705,1.011)$ & 0.065 & $0.860(0.718,1.028)$ & 0.098 & $0.00 \%$ & 0.908 \\
\hline Operative time & 5 & $8,12,14,22,23$ & $-0.188(-0.311,-0.064)$ & 0.003 & $0.119(-0.439,0.677)$ & 0.675 & $94.00 \%$ & 0.000 \\
\hline Blood loss & 3 & $8,22,23$ & $-0.048(-0.184,0.088)$ & 0.490 & $-0.053(-0.202,0.095)$ & 0.481 & $9.50 \%$ & 0.331 \\
\hline LOS & 2 & 12,14 & $0.205(-0.065,0.475)$ & 0.137 & $0.205(-0.065,0.475)$ & 0.137 & $0.00 \%$ & 0.919 \\
\hline 30-day mortality & 2 & 8,23 & $1.330(0.744,2.377)$ & 0.336 & $1.324(0.744,2.356)$ & 0.339 & $0.00 \%$ & 0.908 \\
\hline 90-day mortality & 4 & $12,18,21,22$ & $0.880(0.513,1.511)$ & 0.644 & $0.955(0.569,1.603)$ & 0.861 & $0.00 \%$ & 0.851 \\
\hline
\end{tabular}

RLN, recurrent laryngeal nerve; LOS, length of stay; ESCC, esophageal saqamous cell carcinoma: SMD, STD mean difference; RR, relative risk; Cl confidence Interval: 
A Study

ID

Park, S. (2016)

Deng, H. Y. (2018)

Chen, J (2019)

Zhang, Y. (2019)

$\mathrm{Xu}, \mathrm{Y} .(2020)$

Yang, Y. (2020)

He, Z. F. (2020)

Overall (I-squared $=56.6 \%, p=0.032$ )

NOTE: Weights are from random effects analysis

$$
-.964
$$

SMD $(95 \% \mathrm{Cl})$

Weigh

10.07

10.22

10.72

$-0.01(-0.35,0.33) \quad 12.07$

$0.15(-0.01,0.32) \quad 21.42$

$0.11(-0.06,0.28) \quad 21.06$

$0.25(0.09,0.41) \quad 100.00$

C study

He, H. (2018)

Deng, H. Y. (2018)

Chen, J (2019)

Gong, L. (2020)

$\mathrm{Xu}, \mathrm{Y}$. (2020)

Yang, Y. (2020)

He, Z. F. (2020)

Overall (I-squared $=48.1 \%, p=0.073$ )

$0.11(-0.06,0.28) \quad 30.16$

$0.50(0.21,0.78) \quad 10.37$

$0.17(0.08,0.27) \quad 100.00$
$0.50(0.21,0.78) \quad 14.44$

Study

Deng, H. Y. (2018)

Zhang, Y. (2019)

Xu, Y. (2020)

Overall (I-squared $=0.0 \%, p=0.588$ )

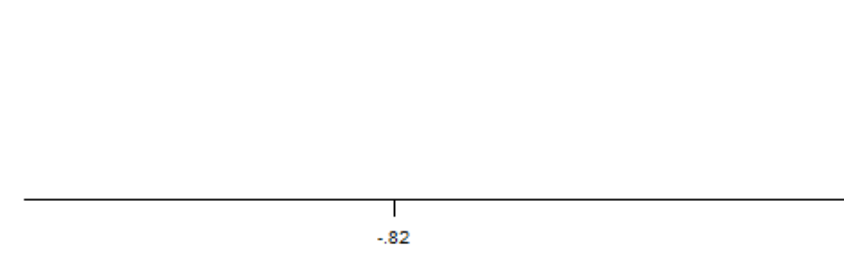

D

Stucy

ID

SMD $(95 \% \mathrm{Cl})$

Deng, H. Y. (2018)

Gong, L. (2020)

Xu, Y. (2020)

Overall (I-squared $=0.0 \%, p=0.480$ )
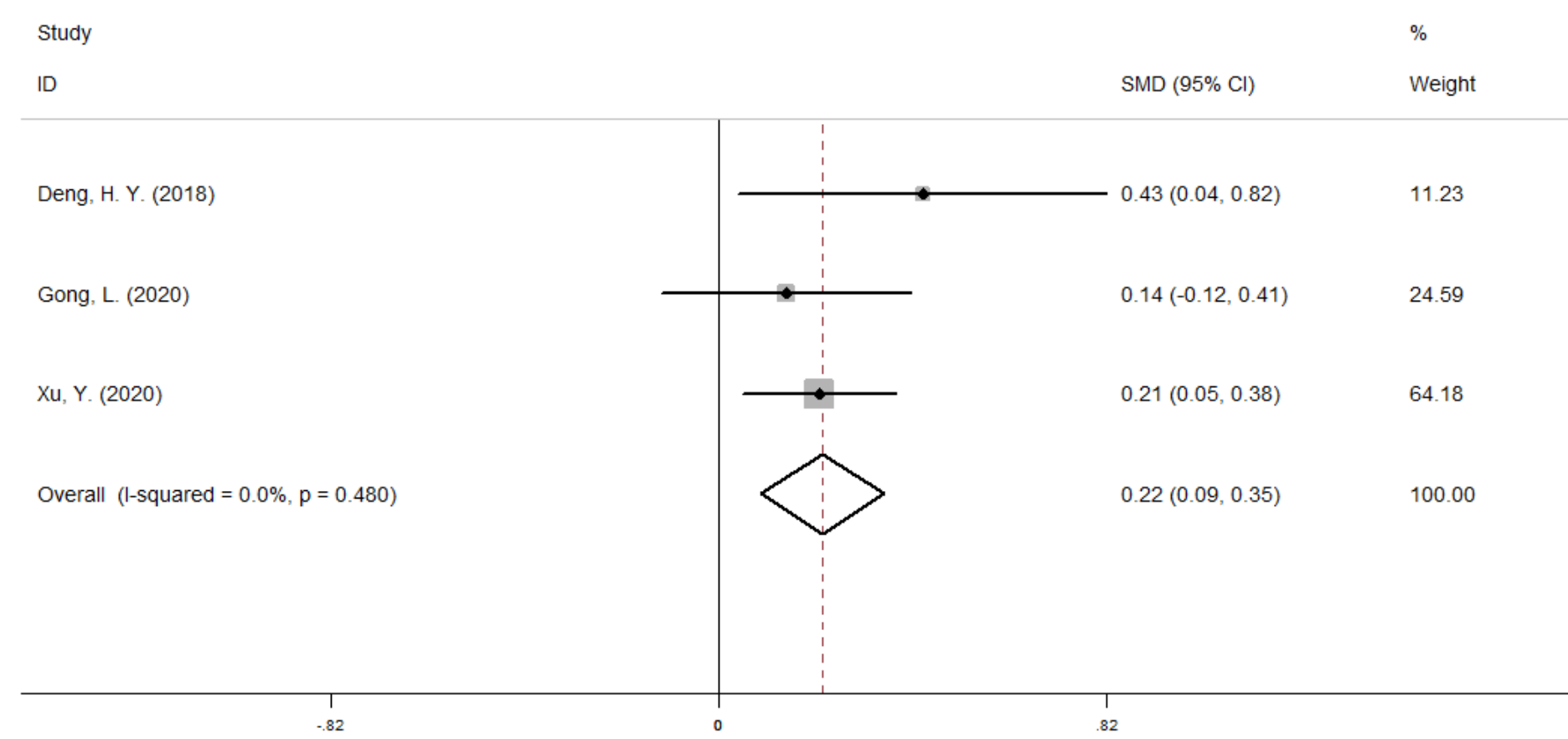

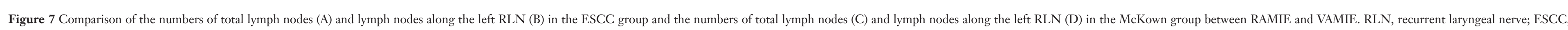
esophageal squamous cell carcinoma; RAMIE, robot-assisted minimally invasive esophagectomy; VAMIE, video-assisted minimally invasive esophagectomy. 
surgical system has been applied to esophagectomy in an increasing number of hospitals. Although a large number of studies have reported the safety and efficiency of RAMIE, the high cost prevents its extensive application. Whether RAMIE can achieve better benefits than VAMIE is particularly important. To our knowledge, this is the third meta-analysis comparing outcomes between RAMIE and VAMIE. Jin et al. (4) reported the first meta-analysis and showed that RAMIE was associated with less estimated blood loss and a lower rate of RLN paresis. Zheng et al. (5) was the second to explore the difference through a meta-analysis and found that RAMIE was associated with a longer operative time and a lower incidence of pneumonia. More high-quality studies have subsequently been published. This meta-analysis enrolled 18 RCSs and 1 random control trial, which was published recently. We found that RAMIE was associated with higher numbers of total lymph nodes harvested and lymph nodes harvested along the left RLN and a lower incidence of pneumonia. No statistically significant difference between the two techniques was observed regarding the numbers of thoracic lymph nodes and lymph nodes along the right RLN, RLN paresis, anastomotic leakage, chylothorax, operative time, blood loss, length of stay (LOS), 30-day mortality or 90-day mortality. Vocal cord palsy is related to the extent of lymph node excision along the RLN.

The Da Vinci surgical system has more technical advantages. Its monitor and equipment are oriented in the same direction, which is beneficial to natural handeye coordination. The surgeon can adjust the camera lens to obtain a suitable surgical field without the aid of an assistant. The three-dimensional self-controlled magnified view allows better visualization of the upper mediastinum. Special equipment with 7 degrees of freedom provides surgical assistance in a limited anatomical space. The system can filter out tremors and synchronize with surgeons' movements to a certain extent, which can ensure stable and meticulous operation. Surgeons can be more focused without standing for a long time. However, a lack of haptic feedback complicates distinction of different organs based on hardness. The surgeon leading the team performs the operation using a console that is not located on the operating table and must address emergencies after meeting sterile requirements.

Lymphadenectomy is an important part of esophagectomy. Lymph node excision, especially along the bilateral RLNs, is crucial for both accurate staging and local control and can be expected to improve clinical outcomes in both ESCC and esophageal adenocarcinoma (30). Skeletonization of the RLN is facilitated by robotics given the articulated and non-tremulous arms. However, careful avoidance of thermal damage and transient traction to the RLN is required to prevent RLN paresis (31). This meta-analysis showed that RAMIE was associated with higher numbers of total lymph nodes harvested and lymph nodes harvested along the left RLN. This result persisted after subgroup analyses based on surgical methods and pathology.

Jin found that RAMIE was associated with a lower incidence of vocal cord palsy based on the finding that the difference in the number of lymph nodes harvested was not statistically significant. Zheng found a similar result when comparing the number of harvested lymph nodes. This meta-analysis indicated that in RAMIE patients, a higher number of lymph nodes along the left RLN were removed. However, no difference in the incidence of vocal cord palsy was found between RAMIE and VAMIE, which we speculated was because surgeons previously emphasized reducing the incidence of complications, but surgeons currently focus on removing a higher number of lymph nodes along the left RLN to improve prognosis.

Zheng et al. found that the operative time for RAMIE was significantly longer than that for VAMIE. The potential reasons may be as follows: (I) an assistant must reposition the robotic cart twice during each operation, and (II) surgeons were unfamiliar with RAMIE. Our results confirmed that the operative time would be shorter according to the learning curve with the development of robotic techniques and proficiency. No significant difference in blood loss or LOS was noted.

This meta-analysis found that RAMIE was associated with a lower incidence of pneumonia than VAMIE. However, we could not reach this conclusion in the McKown group and ESCC group. No differences in RLN paresis, anastomotic leakage or chylothorax were found between the two techniques. The 30-day mortality and 90day mortality showed no significant difference.

Two studies $(25,26)$ were excluded due to data duplication. The treatment center published more than one article with overlapping patients. Motoyama (32) found that RAMIE could reduce the incidence of recurrence at the surgical site. Long-term outcomes, such as overall survival (OS) and disease-free survival (DFS), must be compared between RAMIE and VAMIE. Some complications, such as necrosis and TEF, should be considered. However, these 
complications have rarely been compared between RAMIE and VAMIE due to their low incidence rates. As more patients receive RAMIE, the incidence rates of necrosis and TEF must be evaluated in the future. More large-scale clinical studies, such as the study by Yang et al. (33) are urgently needed to compare these two techniques.

\section{Conclusions}

This study indicated that RAMIE and VAMIE had similar effects and safety. The main advantage of RAMIE is a greater number of harvested lymph nodes, which may be beneficial to diagnosis and local control. RCTs with larger sample sizes and studies reporting long-term outcomes are needed to evaluate the advantages and disadvantages of RAMIE and VAMIE.

\section{Acknowledgments}

Funding: None.

\section{Footnote}

Reporting Checklist: The authors have completed the PRISMA reporting checklist. Available at https://dx.doi. org/10.21037/tcr-21-1482

Peer Review File: Available at https://dx.doi.org/10.21037/ tcr-21-1482

Conflicts of Interest: All authors have completed the ICMJE uniform disclosure form (available at https://dx.doi. org/10.21037/tcr-21-1482). The authors have no conflicts of interest to declare.

Ethical Statement: The authors are accountable for all aspects of the work in ensuring that questions related to the accuracy or integrity of any part of the work are appropriately investigated and resolved.

Open Access Statement: This is an Open Access article distributed in accordance with the Creative Commons Attribution-NonCommercial-NoDerivs 4.0 International License (CC BY-NC-ND 4.0), which permits the noncommercial replication and distribution of the article with the strict proviso that no changes or edits are made and the original work is properly cited (including links to both the formal publication through the relevant
DOI and the license). See: https://creativecommons.org/ licenses/by-nc-nd/4.0/.

\section{References}

1. Bray F, Ferlay J, Soerjomataram I, et al. Global cancer statistics 2018: GLOBOCAN estimates of incidence and mortality worldwide for 36 cancers in 185 countries. CA Cancer J Clin 2018;68:394-424.

2. Guo W, Ma X, Yang S, et al. Combined thoracoscopiclaparoscopic esophagectomy versus open esophagectomy: a meta-analysis of outcomes. Surg Endosc 2016;30:3873-81.

3. Yang Y, Li B, Hua R, et al. Assessment of Quality Outcomes and Learning Curve for Robot-Assisted Minimally Invasive McKeown Esophagectomy. Ann Surg Oncol 2021;28:676-84.

4. Jin D, Yao L, Yu J, et al. Robotic-assisted minimally invasive esophagectomy versus the conventional minimally invasive one: A meta-analysis and systematic review. Int J Med Robot 2019;15:e1988.

5. Zheng C, Li XK, Zhang C, et al. Comparison of shortterm clinical outcomes between robot-assisted minimally invasive esophagectomy and video-assisted minimally invasive esophagectomy: a systematic review and metaanalysis. J Thorac Dis 2021;13:708-19.

6. Suda K, Ishida Y, Kawamura Y, et al. Robot-assisted thoracoscopic lymphadenectomy along the left recurrent laryngeal nerve for esophageal squamous cell carcinoma in the prone position: technical report and short-term outcomes. World J Surg 2012;36:1608-16.

7. Weksler B, Sharma P, Moudgill N, et al. Robot-assisted minimally invasive esophagectomy is equivalent to thoracoscopic minimally invasive esophagectomy. Dis Esophagus 2012;25:403-9.

8. Park S, Hwang Y, Lee HJ, et al. Comparison of robotassisted esophagectomy and thoracoscopic esophagectomy in esophageal squamous cell carcinoma. J Thorac Dis 2016;8:2853-61.

9. Yerokun BA, Sun Z, Yang CJ, et al. Minimally Invasive Versus Open Esophagectomy for Esophageal Cancer: A Population-Based Analysis. Ann Thorac Surg 2016;102:416-23.

10. Weksler B, Sullivan JL. Survival After Esophagectomy: A Propensity-Matched Study of Different Surgical Approaches. Ann Thorac Surg 2017;104:1138-46.

11. Deng HY, Luo J, Li SX, et al. Does robot-assisted minimally invasive esophagectomy really have the 
advantage of lymphadenectomy over video-assisted minimally invasive esophagectomy in treating esophageal squamous cell carcinoma? A propensity score-matched analysis based on short-term outcomes. Dis Esophagus 2019;32:doy110.

12. Grimminger PP, Tagkalos E, Hadzijusufovic E, et al. Change from Hybrid to Fully Minimally Invasive and Robotic Esophagectomy is Possible without Compromises. Thorac Cardiovasc Surg 2019;67:589-96.

13. He H, Wu Q, Wang Z, et al. Short-term outcomes of robot-assisted minimally invasive esophagectomy for esophageal cancer: a propensity score matched analysis. J Cardiothorac Surg 2018;13:52.

14. Chen J, Liu Q, Zhang X, et al. Comparisons of short-term outcomes between robot-assisted and thoraco-laparoscopic esophagectomy with extended two-field lymph node dissection for resectable thoracic esophageal squamous cell carcinoma. J Thorac Dis 2019;11:3874-80.

15. Espinoza-Mercado F, Imai TA, Borgella JD, et al. Does the Approach Matter? Comparing Survival in Robotic, Minimally Invasive, and Open Esophagectomies. Ann Thorac Surg 2019;107:378-85.

16. Motoyama S, Sato Y, Wakita A, et al. Extensive Lymph Node Dissection Around the Left Laryngeal Nerve Achieved With Robot-assisted Thoracoscopic Esophagectomy. Anticancer Res 2019;39:1337-42.

17. Washington K, Watkins JR, Jay J, et al. Oncologic Resection in Laparoscopic Versus Robotic Transhiatal Esophagectomy. JSLS 2019;23:e2019.

18. Zhang Y, Han Y, Gan Q, et al. Early Outcomes of RobotAssisted Versus Thoracoscopic-Assisted Ivor Lewis Esophagectomy for Esophageal Cancer: A Propensity Score-Matched Study. Ann Surg Oncol 2019;26:1284-91.

19. Chao YK, Wen YW, Chuang WY, et al. Transition from video-assisted thoracoscopic to robotic esophagectomy: a single surgeon's experience. Dis Esophagus 2020;33:doz033.

20. Gong L, Jiang H, Yue J, et al. Comparison of the shortterm outcomes of robot-assisted minimally invasive, videoassisted minimally invasive, and open esophagectomy. J Thorac Dis 2020;12:916-24.

21. He ZF, Zheng TL, Liu DL, et al. Comparison of shortterm and long-term efficacy between robot-assisted and thoracoscopy-laparoscopy-assisted radical esophageal cancer surgery. Zhonghua Wei Chang Wai Ke Za Zhi 2020;23:390-5.

22. Xu Y, Li XK, Cong ZZ, et al. Long-term outcomes of robotic-assisted versus thoraco-laparoscopic McKeown esophagectomy for esophageal cancer: a propensity scorematched study. Dis Esophagus 2021;34:doaa114.

23. Yang Y, Zhang X, Li B, et al. Short- and mid-term outcomes of robotic versus thoraco-laparoscopic McKeown esophagectomy for squamous cell esophageal cancer: a propensity score-matched study. Dis Esophagus 2020;33:doz080.

24. Balasubramanian S, Chittawadagi B, Misra S, et al. Propensity matched analysis of short term oncological and perioperative outcomes following robotic and thoracolaparoscopic esophagectomy for carcinoma esophagus- the first Indian experience. J Robot Surg 2021. [Epub ahead of print].

25. Chao YK, Hsieh MJ, Liu YH, et al. Lymph Node Evaluation in Robot-Assisted Versus Video-Assisted Thoracoscopic Esophagectomy for Esophageal Squamous Cell Carcinoma: A Propensity-Matched Analysis. World J Surg 2018;42:590-8.

26. Deng HY, Huang WX, Li G, et al. Comparison of shortterm outcomes between robot-assisted minimally invasive esophagectomy and video-assisted minimally invasive esophagectomy in treating middle thoracic esophageal cancer. Dis Esophagus 2018.

27. Yun JK, Chong BK, Kim HJ, et al. Comparative outcomes of robot-assisted minimally invasive versus open esophagectomy in patients with esophageal squamous cell carcinoma: a propensity score-weighted analysis. Dis Esophagus 2020;33:doz071.

28. Mehdorn AS, Möller T, Franke F, et al. Long-Term, Health-Related Quality of Life after Open and RobotAssisted Ivor-Lewis Procedures-A Propensity ScoreMatched Study. J Clin Med 2020;9:3513.

29. Halpern AL, Friedman C, Torphy RJ, et al. Conversion to open surgery during minimally invasive esophagectomy portends worse short-term outcomes: an analysis of the National Cancer Database. Surg Endosc 2020;34:3470-8.

30. Oshikiri T, Takiguchi G, Urakawa N, et al. Novel "Modified Bascule Method" for Lymphadenectomy Along the Left Recurrent Laryngeal Nerve During RobotAssisted Minimally Invasive Esophagectomy. Ann Surg Oncol 2021;28:4918-27.

31. Hosoda K, Niihara M, Ushiku H, et al. Prevention of intra-thoracic recurrent laryngeal nerve injury with robot-assisted esophagectomy. Langenbecks Arch Surg 2020;405:533-40.

32. Motoyama S, Sato Y, Wakita A, et al. Lower local recurrence rate after robot-assisted thoracoscopic esophagectomy than conventional thoracoscopic surgery 
for esophageal cancer. Sci Rep 2021;11:6774.

33. Yang $Y$, Zhang X, Li B, et al. Robot-assisted esophagectomy (RAE) versus conventional minimally invasive esophagectomy (MIE) for resectable esophageal squamous cell carcinoma: protocol for a multicenter prospective randomized controlled trial (RAMIE trial, robot-assisted minimally invasive Esophagectomy). BMC Cancer 2019;19:608.

Cite this article as: Chen H, Liu Y, Peng H, Wang R, Wang K, Li D. Robot-assisted minimally invasive esophagectomy versus video-assisted minimally invasive esophagectomy: a systematic review and meta-analysis. Transl Cancer Res 2021;10(11):46014616. doi: $10.21037 / \mathrm{tcr}-21-1482$ 